\title{
The hidden link between supercontraction and mechanical behavior of spider silks
}

\author{
Manuel Elices*, Gustavo R. Plaza, José Pérez-Rigueiro, Gustavo V. Guinea \\ Departamento de Ciencia de Materiales, ETSI Caminos, Canales y Puertos. Universidad Politécnica de Madrid, 28040 Madrid, Spain
}

Keywords:

Silk fibers

Fibers processing

Mechanical properties

Biomimetics
A B S T R A C T

The remarkable properties of spider silks have stimulated an increasing interest in understanding the roles of their composition and processing, as well as in the massproduction of these fibers. Previously, the vanability in the mechanical properties of natural silk fibers was a major drawback in the elucidation of their behavior, but the authors have found that supercontraction of these fibers allows one to characterize and reproduce the whole range of tensile properties in a consistent way. The purpose of this review is to summarize these findings.

After a review of the pertinent mechanical properties, the role of supercontraction in recovering and tailoning the tensile properties is explained, together with an alignment parameter to characterize silk fibers. The concept of the existence of a mechanical ground state is also mentioned. These behaviors can be modeled, and two such models - at the molecular and macroscopic levels - are briefly outlined. Finally, the assessment of the existence of supercontraction in bio-inspired fibers is considered, as this property may have significant consequences in the design and production of artificial fibers. 


\section{Introduction}

The unusual combination of high tensile strength and elongation at rupture - that yields the largest work of fracture of any material either natural or artificial (Kaplan et al., 1991, 1994; Vollrath and Knight, 2001) - of spider silks has spurred the interest of researchers to understand, copy or even improve such a remarkable property. Furthermore, spider silk is endowed with a further outstanding feature: the ability to tune its mechanical behavior in a predictable and controllable way (Work, 1976; Denny, 1976; Garrido et al., 2002a,b; Pérez-Rigueiro et al., 2005). Note that as the silk composition is always the same when the spider is tuning the fibers, processing must play a pivotal role in adjusting the final mechanical properties. Composition and processing are two sides of the same coin, and it is obvious that an adequate understanding of both would be of invaluable guidance in the production of artificial bio-inspired fibers.

Another remarkable property of some spider silks is supercontraction (Work, 1977, 1981; Lewis, 1992). This attribute consists of a significant reduction in the length of unrestrained fibers when they are immersed in water or in highly humid environments (Plaza et al., 2006). Although it was not acknowledged immediately, one of the deepest consequences of supercontraction is that it reflects the existence of a ground state (Pérez-Rigueiro et al., 2003). This basic, or initial, state can be recovered regardless of the fiber loading history by simply subjecting the unrestrained fiber to water immersion and subsequent drying (Elices et al., 2004). It has also been found that the whole range of tensile properties exhibited by these fibers - including naturally spun and forced silked fibers - can be obtained in a reproducible way by simply stretching the fibers in water from the ground state (Guinea et al., 2005b).

The purpose of this review is to summarize the work undertaken by the authors in looking for a link between supercontraction and mechanical properties. Most of the experimentation was performed with the silk fibers from the major ampullate gland of the spider Argiope trifasciata but, in the authors' opinion, the results are generalizable to draglines of other spiders as well as to other silks.

The paper is structured as follows: First, some mechanical properties of the spider silk fiber, as stress-strain curves, tenacity or its versatility to tune its behavior, are reviewed in Section 2. The role of controlled supercontraction in tailoring the mechanical behavior of silk fibers is considered in Section 3. Two models at micro and macro levels, able to reproduce the mechanical response of the spider silk fibers as well as the effect of water on the stress-strain curves, are outlined in Section 4. Finally, the presence of supercontraction and the existence of a ground state in artificial silk fibers are explored in regenerated silkworm fibers and in bio-inspired fibers in Section 5. The assessment of a ground state and its link with supercontraction should have significant implications for the design and production of silk fibers.

\section{Some remarkable properties of spider silks}

A. trifasciata spiders produce different kinds of silk fibers for various functions such as web building, prey immobilization,

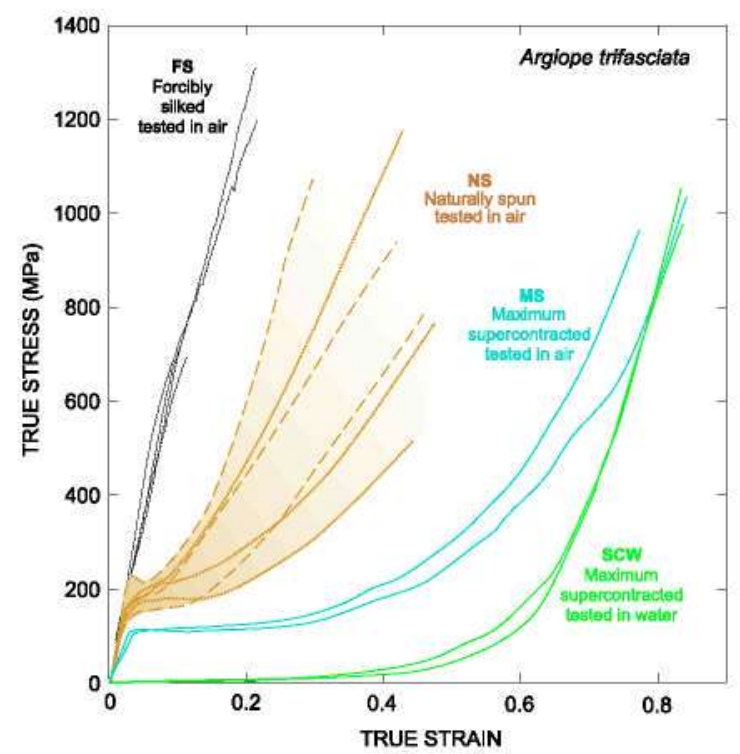

Fig. 1 - Stress-strain curves from MA gland silk of Argiope trifasciata. NS fibers were retrieved either from the web or from the safety line. All tests in air were performed at $20{ }^{\circ} \mathrm{C}, 35 \% \mathrm{RH}$, and a strain rate of $0.0002 \mathrm{~s}^{-1}$.

or cocoon silk. This article will concentrate on the strongest of the fibers produced, those used as the dragline and for web frames and radii, which are spun by the major ampullate (MA) gland (Elices et al., 2005).

Among the interesting properties of MA silk, three will be briefly reviewed in this section; (i) versatility to be tuned according to spider needs (the spider may be able to tailor the tensile behavior of the MA silk to match its intended use by varying the fiber diameter and microstructure); (ii) remarkable tenacity, which results in the largest work to fracture of any material, either natural or artificial, and (iii) capacity to supercontract when immersed in water or subjected to high relative humidity environments with its ends unrestrained.

\subsection{The effect of spinning forces}

The MA silk fibers from A. trifasciata can be classified into two groups according to the collection procedure: those spun during the building of the web (radii, frame, and mooring lines), as well as the safety line, will be labeled as naturally spun (NS), and those obtained from an immobilized spider by forced silking will be labeled forcibly silked (FS).

The tensile properties of NS fibers obtained directly from the web show a wide scatter of the stress-strain curves, as illustrated in Fig. 1, which can be attributed in part to the intrinsic variability of biological materials (Pérez-Rigueiro et al., 2001). The flow of material through the spinning apparatus is under the active control of the spider (Vollrath and Knight, 2001) which changes the fiber diameter and microstructure. It was also observed that the stress-strain characteristics of a dragline produced in an undisturbed vertical climb are more reproducible than those of silk spun during horizontal crawling (Garrido et al., 2002a). From an experimental point of view, the variability in the properties 
Table 1 - Average values of mechanical properties of silk fibers and high-performance synthetic fibers.

\begin{tabular}{|c|c|c|c|c|c|}
\hline \multicolumn{2}{|c|}{ Fiber type } & Tensile stress (MPa) & Breaking strain (\%) & Resilience $(\mathrm{M}) / \mathrm{m}^{3}$ ) & Reference \\
\hline \multicolumn{6}{|l|}{ Silk fibers } \\
\hline \multicolumn{6}{|l|}{ Argiope trifasciata } \\
\hline Naturally spun & NS & 780 & 26 & 90 & Pérez-Rigueiro et al., 2001 \\
\hline Forced silked & FS & 1110 & 17 & 110 & \\
\hline Max. & MS & 990 & 81 & 240 & Elices et al., 2009 \\
\hline \multicolumn{6}{|l|}{ supercontracted } \\
\hline \multicolumn{6}{|l|}{ Nephila inaurata } \\
\hline Forced silked & FS & 1800 & 26 & 240 & \\
\hline Max. & MS & 1480 & 69 & 270 & \\
\hline \multicolumn{6}{|l|}{ supercontracted } \\
\hline \multicolumn{6}{|l|}{ Nephila clavipes } \\
\hline Forced silked & FS & 1200 & 17 & 110 & Swanson et al., 2006 \\
\hline \multicolumn{6}{|l|}{ Bombix mori } \\
\hline Forced silked & FS & 730 & 11 & 50 & Pérez-Rigueiro et al., 2000 \\
\hline Commercial & & 600 & 23 & 90 & Weidmann et al., 1990 \\
\hline \multicolumn{6}{|l|}{ Synthetic fibers } \\
\hline PBO & & 5800 & 2.5 & 70 & \\
\hline Kevlar 49 & & 3000 & 2.7 & 50 & Weidmann et al, 1990 \\
\hline Nylon $6,6(\mathrm{HT})$ & & 750 & 18 & 70 & Weidmann et al., 1990 \\
\hline PET (HT) & & 800 & 7 & 30 & Weidmann et al, 1990 \\
\hline \multicolumn{6}{|l|}{ Steel } \\
\hline Strand & & 1800 & 5 & 70 & Weidmann et al., 1990 \\
\hline Piano wire & & 3000 & 1.5 & 25 & Weidmann et al., 1990 \\
\hline
\end{tabular}

of NS silk has been a major drawback since it has prevented the drawing of reliable conclusions (Madsen et al., 1999).

In contrast, FS fibers show acceptable reproducibility if some precautions are taken (Guinea et al., 2005a), as is apparent in Fig. 1. The tensile properties of FS fibers have been found to differ considerably from those of NS fibers: FS fibers are stiffer and show a smaller deformation at breaking than NS. Since both types have the same protein composition, the differences are highly likely to be the result of changes in the course of processing.

The forced silking technique allows the researcher to measure the silking force and retrieve the sample to evaluate its tensile properties. A correlation between silking stress and tensile properties has been verified and published elsewhere (Pérez-Rigueiro et al., 2005). The silking stress greatly influences the type of silk fiber: when the stress is high, i.e. around the conventional yield limit, the fibers are stiffer than those naturally spun by spiders. As the stress decreases, fiber stiffness decreases, and forcibly silked fibers come to resemble the naturally spun ones. At very low silking stresses, the fiber is even more compliant, and its behavior is similar to that of the supercontracted fiber tested in air (see Fig. 1 and comments below on supercontraction). These results suggest that the tensile behavior of MA silk fibers can be modulated through the silking stress in a wide range that spans from maximally supercontracted fibers to stiff silked fibers, including naturally spun fibers produced during web building and also as safety lines.

\subsection{Tenacity of major ampullate silk fibers}

The tensile strength of some MA silk fibers is as high as the steel used for suspension bridges and for prestressing concrete, and of the same order of magnitude as artificial high performance fibers, such as Kevlar or PBO. In contrast, however, these spider fibers are characterized by a very high strain at break, of the order of $20 \%-30 \%$ (see Table 1 ).

Such an unusual combination of high strength and elongability leads to a high capacity for absorbing energy also called tenacity or resilience - which can be quantified by the area under the stress-strain curve measured in a tensile test. Spider silk tenacity has not been achieved in synthetic high performance polymeric fibers, as shown in Table 1. In man-made fibers, indeed, any improvement in tensile strength is always associated with a decrease in the strain at break and vice-versa. As a result, the exceptional tenacity of MA silk fibers is a remarkable property.

This ability to store energy enables spiders to intercept and catch their prey, absorbing their kinetic energy. Undoubtedly, selective evolution pressure must have contributed to the high tenacity of MA silks in orbicular spiders; however, to make artificial fibers inspired by spider silks a reliable relationship between properties and composition on the one hand, and processing on the other, needs to be discerned. Processing has an influence, as previously mentioned when dealing with the role of the spinning force in the tensile behavior. Composition also affects the tensile behavior; correlations have been drawn between an alamine-rich "crystalline module" and the tensile strength, and between a proline-containing "elasticity module" and extensibility (Hayashi et al., 1999). Both aspects, composition and processing, are involved in this outstanding property.

\subsection{Supercontraction}

Another intriguing behavior of spider silk fibers is supercontraction. The term supercontraction refers to the shrinkage up to half its initial length - of some silk fibers (Work, 1977. 


\begin{tabular}{|c|c|c|}
\hline Fiber type & $S C$ index $\left(L_{0}-L_{C}\right) / L_{0}$ & Reference \\
\hline \multicolumn{3}{|l|}{ Argiope trifasciata } \\
\hline Dragline (naturally spun) & 0.31 & Pérez-Rigueiro et al., 2003 \\
\hline Dragline (forced silked) & 0.52 & Elices et al., 2009 \\
\hline \multicolumn{3}{|l|}{ Nephila sp } \\
\hline Dragline (naturally spun) & 0.20 & Work, 1985 \\
\hline \multirow[t]{3}{*}{ Dragline (forced silked) } & 0.40 & Elices et al., 2009 \\
\hline & 0.40 & Liu et al., 2005 \\
\hline & 0.44 & Jelinski et al., 1999 \\
\hline
\end{tabular}

1981; Vollrath, 1992) when immersed in water in an unrestrained condition. Measurement of supercontraction is described elsewhere (Elices et al., 2004). The supercontraction index, $S C$, is defined as $\left(L_{0}-L_{c}\right) / L_{0}$, where $L_{0}$ is the initial fiber length and $L_{c}$ the length of the supercontracted unstressed fiber. Table 2 shows values of the SC index for selected spider silk fibers. Note that the SC index depends on the fiber type; naturally spun fibers that show a wide span in the stress-strain curves (as illustrated in Fig. 1) also show a range of values for the SC index (in Table 2 only average values are recorded). Forced silked fibers, on the contrary, are more similar, and the same happens with the SC index. (The values shown in Table 2 for Nephila are almost the same, in spite of having been measured by three independent research groups.)

Furthermore, it was also found that supercontraction leads to another significant modification of the properties of spider silk fibers (Gosline et al., 1984), since the tensile behavior of supercontracted MA silk fibers tested in water corresponds to an elastomer, as illustrated in Fig. 1. The elastomeric behavior is characterized by an extremely low value of the elastic modulus at small deformations and a significant increase of stiffness at large deformations, in contrast with the behavior of MA silk fibers tested in air, either as spun or subjected to previous supercontraction and dried (Fig. 1).

The absence of a clear biological function for the changes induced in MA silk fibers by supercontraction produced an ongoing debate about its role in the performance of the material. In parallel with its discovery, it was proposed that supercontraction might allow the web to remain taut under high humidity conditions (Work, 1977). This interpretation was apparently questioned by measurements on the relaxation of stresses in MAS fibers under high relative humidity conditions (Bell et al., 2002) that seemed to establish the complete relaxation of supercontraction stresses in a period of minutes. However, later studies (Guinea et al., 2003; Savage et al., 2004) established that typical relaxation times were in the range of hours, and stresses relaxed a relatively small percentage of their initial value. These results indicated that the role of supercontraction in tensioning the web under humid environments could not be rejected on purely mechanical grounds.

\section{Controlled supercontraction tailors the ten- sile behavior}

Supercontraction turns out to be not only a curious property, but also a crucial feature to characterize and reproduce

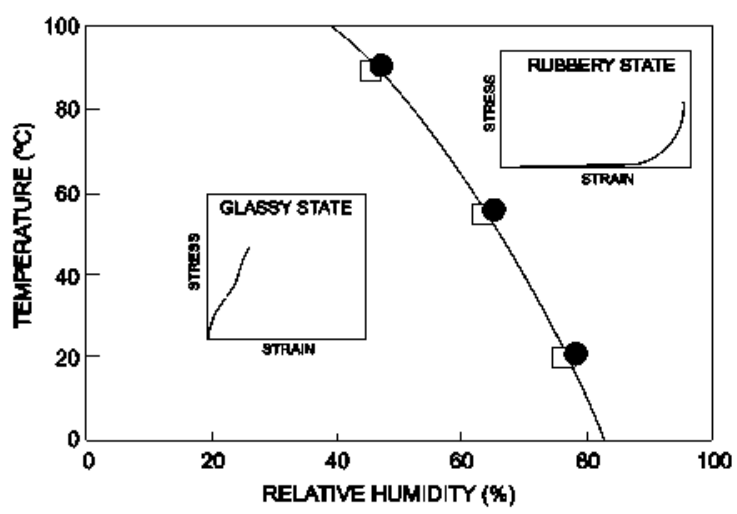

Fig. 2 - Transition from the glassy to the rubbery state as a function of relative humidity and temperature. Transition values of supercontraction index (open squares) and initial elastic modulus (full circles) are indicated. Source: From Plaza et al. (2006).

the whole range of tensile properties exhibited by spider silk through a combined effect of supercontraction and stretching. To assess this far-reaching property, it is necessary to review briefly three aspects: (i) the glassy and rubbery states a spider silk can exhibit; (ii) its capacity to recover the same mechamical state, i.e. that the tensile properties of maximum supercontracted fibers are independent of the previous loading history; (iii) the possibility to tune the mechanical properties of silk fibers in a predictable and reproducible way by a simple procedure; wet stretching. A detailed description of all these aspects is given in the quoted references.

\subsection{The glassy and rubbery states (Plaza et al., 2006)}

Humidity and temperature have a strong influence on the mechanical behavior of spider silk fibers. Tensile tests of spider silk fibers were performed systematically under controlled temperature and humidity. On increasing the temperature or the humidity, the behavior of silk fibers changed from stiff (typical of glassy polymers) to compliant, similar to that of elastomeric fibers.

Compliance can be characterized by means of the initial elastic modulus. At a given temperature, this parameter showed a sudden transition within a small range of values of humidity, a behavior indicative of a glass-rubber transition (Fig. 2).

The supercontraction index also showed a sudden transition within a narrow range of values of humidity in tests 
performed at a constant temperature. Curiously, the average values of temperature and humidity along this transition were very similar to the values of the glass-rubber transition (Fig. 2).

All these results agreed with a model of silk fibers as a semi-crystalline material made of amorphous flexible chains reinforced by crystallites, and provided a plausible explanation of the glass-rubber transition. The chains were strongly hydrogen-bonded and the presence of water and/or high temperature influenced the hydrogen bonding; water is known to have a plasticizing effect by preventing the formation of hydrogen bonds between chains, with temperature working in a similar way.

\subsection{Recovery in spider silk fibers (Elices et al., 2004)}

Research by Gosline et al. (1984) revealed that supercontraction has profound implications on the mechanical behavior of spider silk, even suggesting a relationship between the microstructure and the tensile properties (Termonia, 1994, 2000). We found that supercontraction modified the tensile properties of spider silk and provided a key to understanding some aspects of the fiber tensile behavior.

Representative stress-strain curves of forced silked (FS) fibers, naturally spun (NS) fibers and maximum supercontracted (MS) fibers are shown in Fig. 3(a), which illustrates both the variability of the stress-strain curves and the irreversible effect of large deformations. In addition, two types of curves are depicted: noval stress-strain curves and curves after an unloading, and reloading cycle. Note that the reloading behavior is different from the noval behavior. All of these curves came from the same spider and from the same type of silk, from the major ampullate gland.

Fig. 3(b) shows the stress-strain curves of noval FS and NS fibers before and after maximum supercontraction. Note that the noval curves, which were very different, merge completely after supercontraction.

Fig. 3(c) shows the stress-strain curves of reloaded FS, NS and MS fibers, before and after maximum supercontraction. Again, all three recovered curves merged into the same curve (the maximum supercontracted).

Hence, the tensile properties of maximum supercontracted (MS) fibers are independent of the previous loading history of the fiber (noval or reloaded) and from the collection procedure (natural or forced silked). This state after maximum supercontraction, a type of annealing, can be considered as a kind of "ground state" and the capacity to recover the initial properties after maximum supercontraction is the starting point for making fibers with predictable and reproducible features.

\subsection{Tailoring the tensile properties of spider silk fibers} (Guinea et al., 2005b)

The existence of a ground state allows tuning the properties of MA silk fibers in a predictable and reproducible way by a procedure named wet stretching that consists of stretching a silk fiber in water that has previously been subjected to maximum supercontraction.
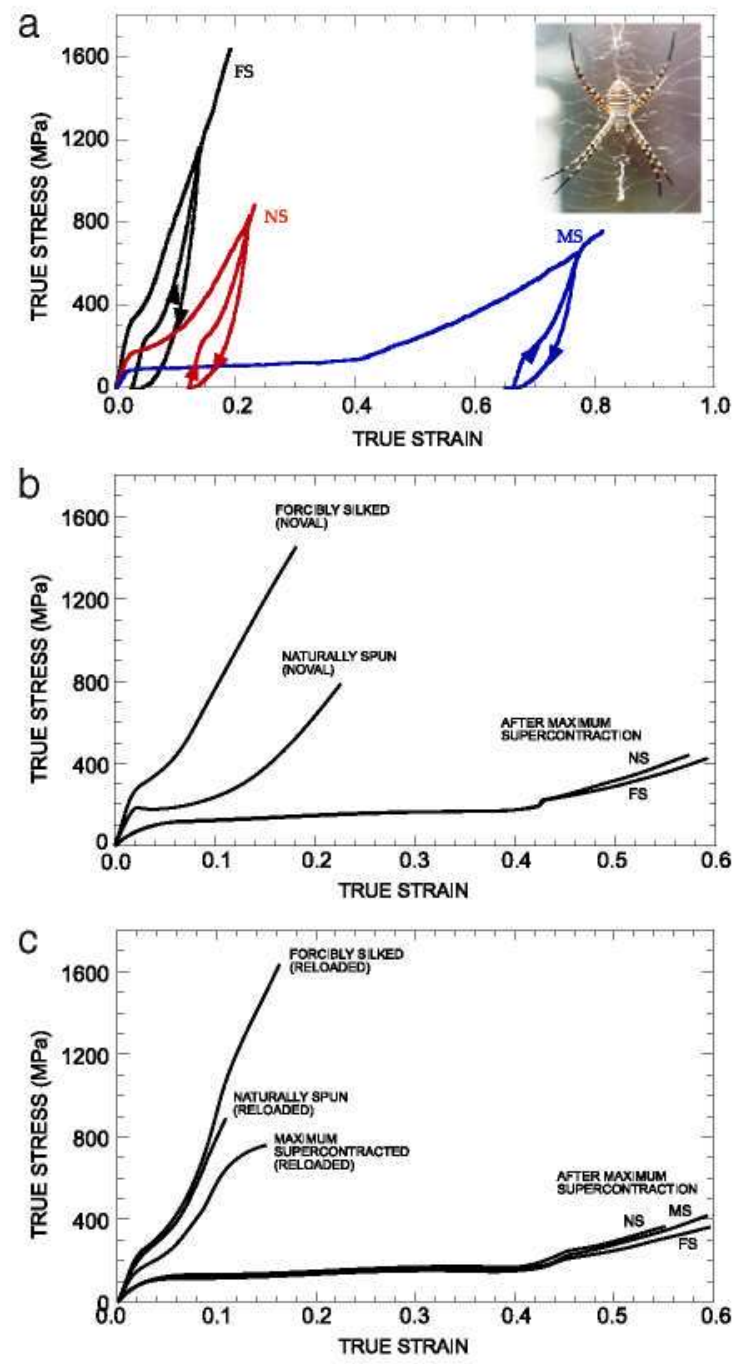

Fig. 3 - (a) Stress-strain curves of forced silked (FS), naturally spun (NS) and after maximum supercontraction (MS) fibers: Noval curves and curves after unloading and reloading cycle (major ampullate silk from Argiope trifasciata, see inset). (b) Stress-strain curves of noval FS and NS fibers before and after maximum supercontraction. (c) Stress-strain curves of reloaded FS, NS and MS fibers before and after maximum supercontraction.

The wet stretching process can be summarized as follows (see Fig. 4): the fiber is allowed to contract unrestrained (this length is called $L_{M S}$ ). Then it is stretched to a selected length $L_{A}$ and the ends are clamped, water is removed and the fiber is dried overnight. Stresses built up in the fiber during this process are relaxed by unloading to the final length, $L_{C}$. Load evolution during the wet stretching is also sketched in Fig. 4. Wet stretching is characterized quantitatively by the alignment parameter $\alpha$, which is defined as $\alpha=L_{C} / L_{M S}-1$.

Fig. 5 shows stress-strain curves obtained with the wet stretching procedure for different values of the alignment parameter $\alpha$, ranging from $\alpha=0$ to $\alpha=1.1$. The maximum supercontracted state - the ground state - corresponds to $\alpha=0$. Forced silked fibers (also shown in the figure) are reproduced with $\alpha=1$.11. In between is the range of natural 

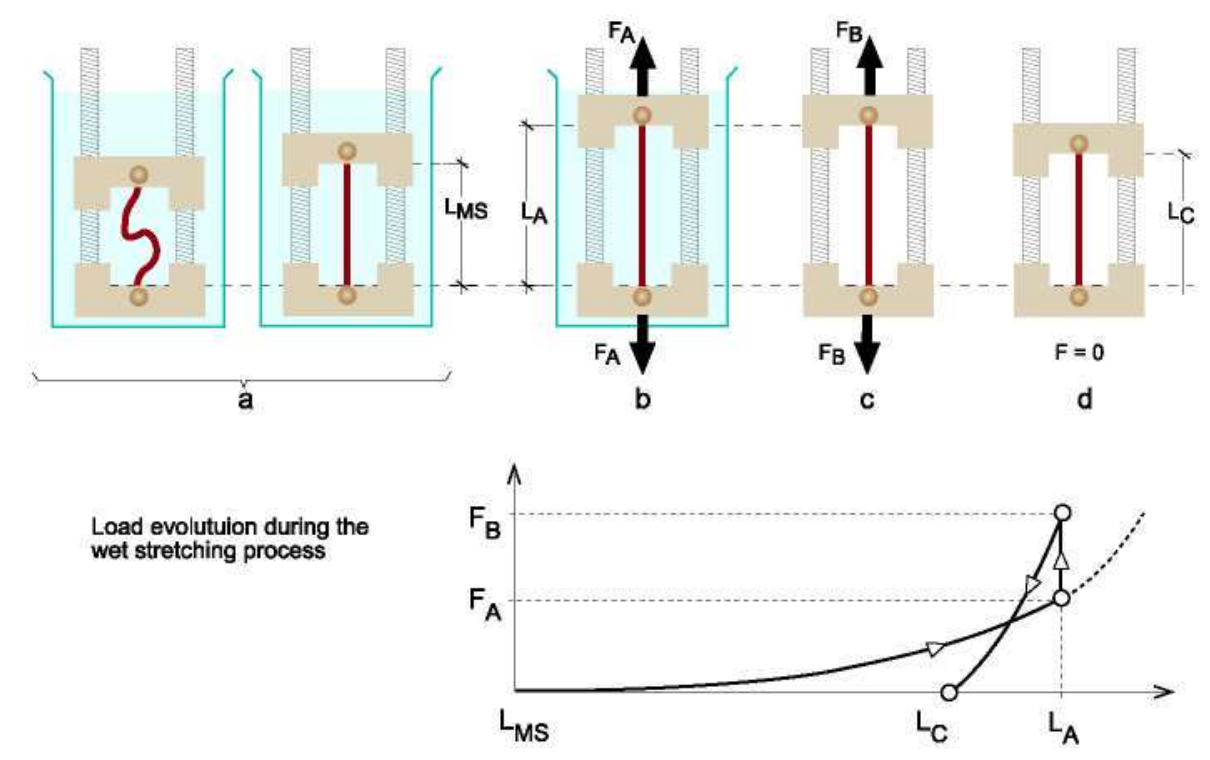

Fig. 4 - Wet stretching procedure: (a) the fiber is allowed to contract unrestrained up to the supercontracted length $L_{M S}$; (b) it is stretched to the selected length $L_{A}$ and the ends clamped in this position; (c) water is removed and the fiber is dried overnight; (d) stresses built up in the fiber during drying are relaxed by unloading down to the final length $L_{C}$.

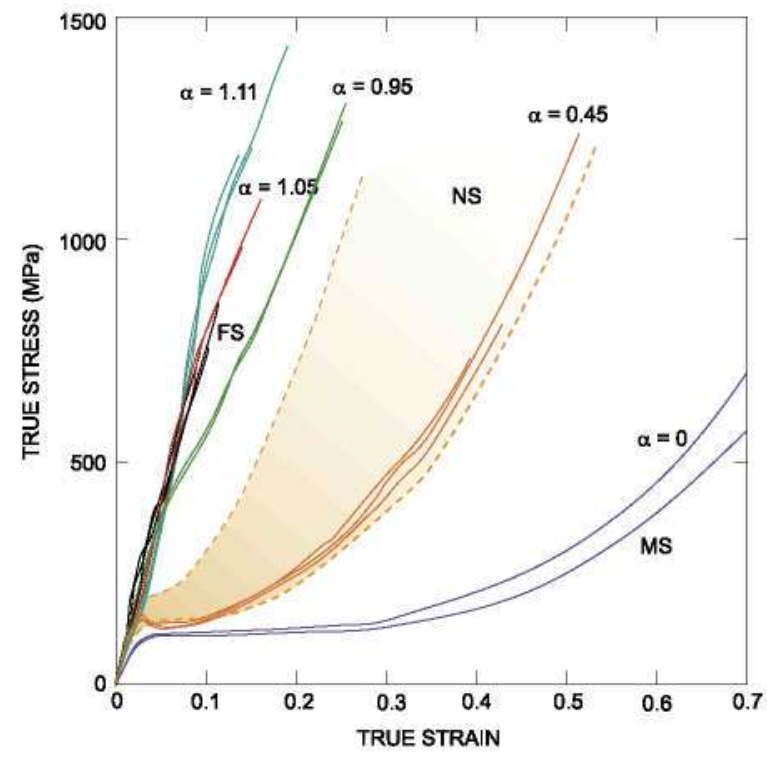

Fig. 5 - Stress-strain curves obtained with the wet stretching procedure, using different values of the alignment parameter $\alpha$. Note that for $\alpha$ close to 1.0, typical forced silked fibers (also shown) are reproduced. The span of natural silked (NS) fibers range between $\alpha=0.45$ and $\alpha=0.90$. The ground state (MS) corresponds to $\alpha=0$.

spun fibers, that can be replicated with values of the alignment parameter between $\alpha=0.45$ and $\alpha=0.90$.

It has been hypothesized that a mechanism similar to wet stretching takes place in the silk gland of the spider, with it being the basis of the production of natural fibers with tunable properties. It is assumed that, immediately after solidification within the gland, the fiber shows the elastomeric behavior of a supercontracted fiber in water, which allows the spider to fix the alignment parameter by controlling the stress exerted on the fiber during spinning (Pérez-Rigueiro et al., 2005).

The results presented above highlight the importance of the existence of a ground state, a property that underlies the shrinkage of the fibers in humid environments, their recovery after irreversible stretching and the tuning of the mechanical properties of MA silk. Besides, the proposed relationship between supercontraction, processing and the possibility of modifying the tensile properties of the fibers sets the question of the biological function of supercontraction under a new perspective. In this regard, supercontraction itself would not be the main property that has driven natural selection during the evolution of orb-web spiders, but most likely the possibility of adapting the material to the requirements of the spider in the web or as a safety line (Elices et al., 2009). In this context, supercontraction can be understood as a byproduct of the existence of a ground state, a hypothesis that does not exclude the argument that tensioning the web under humid environments might be an additional benefit from the underlying property.

\section{Models to account for mechanical behavior}

$\mathrm{Ab}$ initio computations of the mechanical properties of spider silk fibers are still in their infancy, due to the complexity of their large polymeric chains. Even so, several approaches have been devised to cope with this problem: at one extreme there are molecular models which bypass details of the deformation on an atomistic scale and focus instead on a length scale of the order of the distance between entanglements, and at the other are macroscopic models based on the mechanics of continuous media and using constitutive equations that capture the main microstructural features. Examples of both models are briefly summarized here (for specific features see the quoted references). 


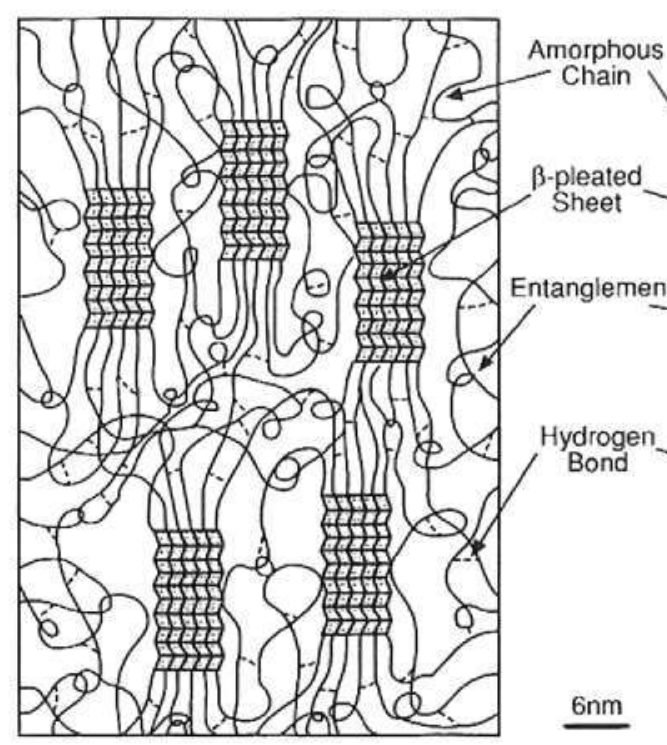

a

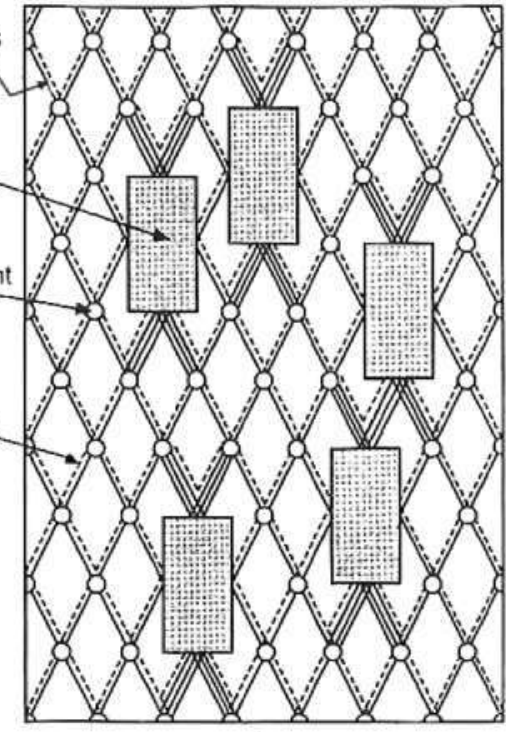

b

Fig. 6 - Molecular model for spider dragline. (a) System of amorphous and crystalline chains. For the purpose of easy representation, the figure is for a $15 \%$ volume fraction of beta-pleated sheets; (b) more schematic representation in which the details of the amorphous chains have been omitted and only end-to-end segments are shown. Individual hydrogen bonds have been replaced by "overall" bonds (dotted lines) connecting every entanglement to its neighbors. The 3-lines segments indicate the high modulus layer in the amorphous phase.

Source: From Termonia (2000).

\subsection{Termonia's molecular model (Termonia, 1994, 2000)}

Spider dragline was identified as a semi-crystalline material made of amorphous flexible chains reinforced by crystallites. The amorphous part was attributed to oligopeptide chains rich in glycine, and the crystallites were believed to be made of hydrophobic polyalanine sequences arranged into betapleated sheets. The chains were strongly hydrogen-bonded.

Termonia's model, based on the above considerations, is depicted in Fig. 6. Initial lattice parameters such as molecular weight, density of entanglements, crystalline fraction, etc. were fixed from experimental data. Silk fibers were modeled as a network where knots were the nanocrystallites or the entanglements and threads the amorphous chains or hydrogen bonds connecting every entanglement to their neighbors. After completion of the network, the deformation of the lattice was performed in a succession of very small length increments. After each strain increment and upon complete relaxation of all lattice sites, individual bonds and entanglement points were visited at random by a Monte-Carlo lottery and four processes allowed to occur:

a. Breaking of the hydrogen bonds.

b. Stretching of the chains in the amorphous regions.

c. Breaking of the chain strands in the amorphous regions.

d. Network relaxation.

After the network of entanglements had been fully relaxed towards mechanical equilibrium, an additional elongation increment was applied to the network and the four processes described above restarted for another time interval. And so on and so forth, until the network failed.

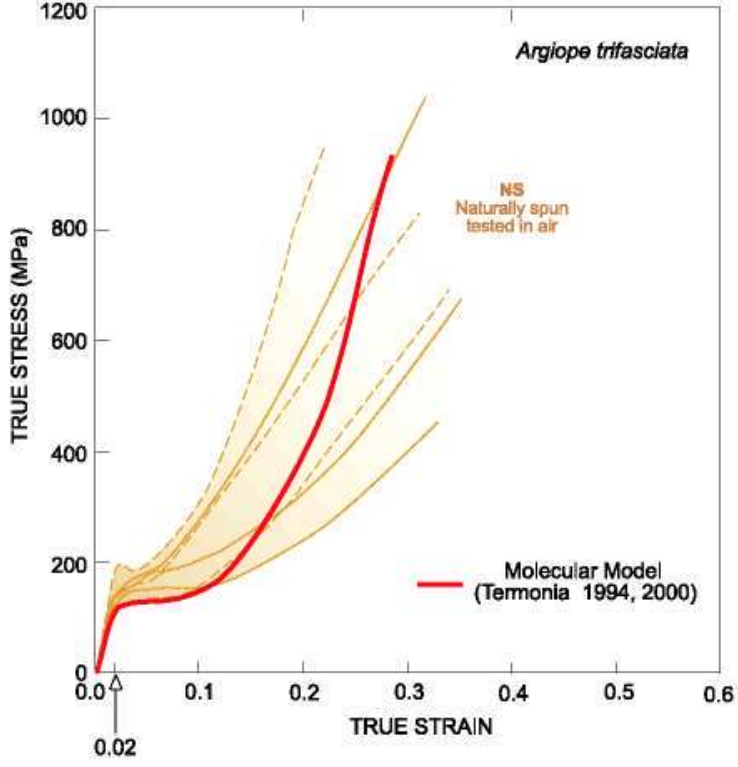

Fig. 7 - Calculated and experimental stress-strain curves for spider dragline silk fibers.

Source: The experimental curves are taken from Elices et al. (2005).

This model was able to make qualitative and quantitative predictions of stress-strain curves, as shown in Fig. 7. Using reasonable initial data (see Termonia, 2000) the model predicted at small strains - under 0.002 - a linear curve with modulus $E=10 \mathrm{GPa}$, in agreement with experimental observations (Gosline et al., 1994; Work, 1977; Elices et al., 2005). 


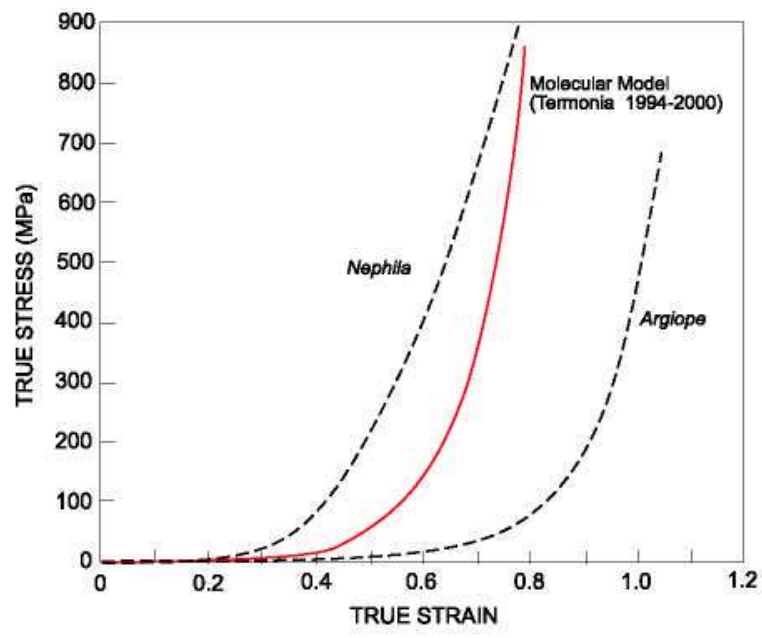

Fig. 8 - Calculated and experimental stress-strain curves for spider dragline silk fiber, first immersed in water and then strained at a rate of $1.42 / \mathrm{min}$.

Source: The experimental curves are taken from Elices et al. (2009).

At about 0.02 strain the hydrogen bonds between chains in the amorphous region started to break, leading to the formation of a yield point at which the stress-strain curve reaches a plateau. At 0.1 strain and higher the hydrogen bond breaking process was almost complete, with the stress resuming its increase with strain. Fracture at strains circa 0.3 occurred, in agreement with most experimental results.

The model also reproduced the effect of water in the stress-strain curves. The model assumed that the only effect of water on the fiber was to prevent the formation of hydrogen bonds between chains in the amorphous phase. Upon stretching the network, now free of hydrogen bonds in the amorphous phase, the stress-strain curve exhibited a rubber-like behavior (Fig. 8). Also represented in this figure are typical experimental curves for wet draglines from two spiders (Elices et al., 2009).

This molecular model reveals the importance of the small crystalline beta-sheets, which created inside the amorphous phase a thin layer with a modulus higher than the bulk. Moreover, it also provides a plausible explanation for the presence of a monodisperse molecular weight distribution, typical of spider fibers (O'Brien et al., 1998), never attained in synthetic man-made fibers (Termonia, 2000).

\subsection{PGE macroscopic model (Planas et al., 2007)}

The objective of this model was to reproduce the mechanical response of the spider silk fiber - regarded as fiber-reinforced material - without dealing with mesoscopic details during every loading step. The spider silk fiber was assumed to consist of sets of microfibrils dispersed into a deformable matrix. It was also assumed that no force was transmitted through the matrix in the composite material (i.e., that the matrix was perfectly deformable and forced only buildup in the microfibrils as a consequence of deformation).

To make the analysis simpler, an equivalent continuum macroscopic model was introduced by forcing the model to

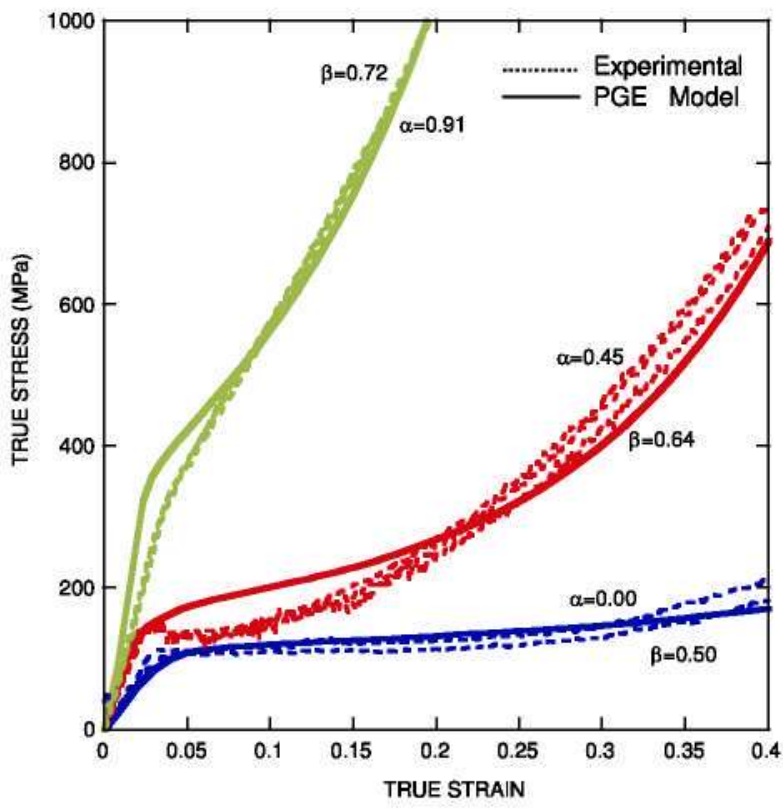

Fig. 9 - Calculated and experimental stress-strain curves for a spider dragline fiber. Model results are labeled by the orientation index $\beta$. Experimental data are identified by the alignment parameter $\alpha$.

Source: From Guinea et al. (2005b).

give the same mechanical dissipation as the fiber-reinforced material (i.e., spider silk fibers) under any arbitrary motion.

This condition had to be complemented with the relationship between the nominal stress and strain within the microfibrils (Planas et al., 2007). For this relationship, a simple model was postulated, capturing the well known behavior of spider silks, a nonlinear spring representing the stretching and orientation of molecular chains in the rubbery state (i.e., with the network of hydrogen bonds deactivated), in parallel with a skidding block with a linear spring to account for the breaking of hydrogen bonds and molecular slippage when the fiber was over the glass transition.

Fig. 9 shows the experimental results of tensile tests performed in air on spider silk fibers (MA) with three different degrees of molecular alignment identified by the alignment parameter $\alpha$, obtained as described above (Guinea et al., 2005b).

In the same figure are depicted the results of numerical computations, using the macroscopic model, for the three kinds of fibers. Each type of fiber is characterized by the orientation index $\beta$ (Planas et al., 2007), which can be interpreted as the ratio between the projected length of microfibrils along the fiber axis and their total length at a given instance. A random orientation of microfibrils gives $\beta=0.5$ whereas $\beta=1$ gives all the microfibrils parallel to the fiber axis.

As shown in the figure, the agreement between experiments and simulation is remarkable, especially taking into account that the numerical modeling reproduces the experimental procedure step by step. The proposed model follows the large deformation of MA silk fibers and properly captures their alignment process, easily incorporating the main operative microstructural mechanisms. In addition, the model 
gives valuable information, such as that of the orientation in$\operatorname{dex} \beta$, which can be useful in linking macroscopic parameters like the alignment parameter $\alpha$ with the internal distribution of microfibrils.

\section{Biomimetic approaches}

The outstanding tensile properties of spider silks have created an increasing interest in mass-production of these fibers. In contrast to silkworms, it is impractical to farm most spiders because of their territorial and cannibalistic nature and one has to resort to biomimetic approaches, such as identification of key protein sequences, production of genetically engineering analogues and spinning the dope.

Natural silk fibers are the outcome of a balanced combination between carefully tailored compositions and highly sophisticated spinning glandular systems (Vollrath and Knight, 2001, Gosline et al., 1999, Viney, 2000). Both aspects, composition and processing, have to be kept in mind when dealing with artificial fibers. To this end, processing can be investigated by spinning a dope of dissolved silk, where the composition is the same as the natural one. On the other hand, the composition can be evaluated by spinning dopes of spider silk-like proteins that are based on sequences of dragline silk proteins. These two approaches will be considered later.

The purpose of this section is not to review such approaches, recently several excellent surveys have been published (Hardy et al., 2008; Kluge et al., 2008; Heim et al., 2009, among others and references therein), but to focus on the assessment of the existence of supercontraction in bioinspired fibers, as this property has important consequences in the design and production of artificial fibers that mimic major ampullate gland spider silks.

\subsection{Processing of regenerated silk}

Regenerated silk fibers are obtained by spinning dopes of natural dissolved silk and consequently have a composition closer to that of natural ones. Differences between natural and regenerated fibers should rely more on the spinning process than on composition.

Seidel et al. (2000) investigated the mechanical performance of regenerated silk fibers from the spider Nephila clavipes. They found that the as-spun fibers are very weak, but both the strength and stiffness of the regenerated silk can be enhanced by two orders of magnitude by post spinning drawing. The tensile strengths and moduli of fibers obtained under the same spinning conditions show an exponential dependence on the diameter of the processed specimen. Also, they revealed that the fraction of alanine residues adopting the beta-sheet conformation, the extent of its crystallinity and its overall degree of molecular orientation, influence the tensile properties of regenerated silk. All these parameters are determined by the post spinning processing history of the sample, especially by the extent of draw applied to the fiber.

Shao et al. (2003) reported tests performed with fibers from regenerated silk from Nephila edulis. Here no spinning was performed; instead filaments were pulled away from a dilute aqueous solution of spider major ampullate silk protein.

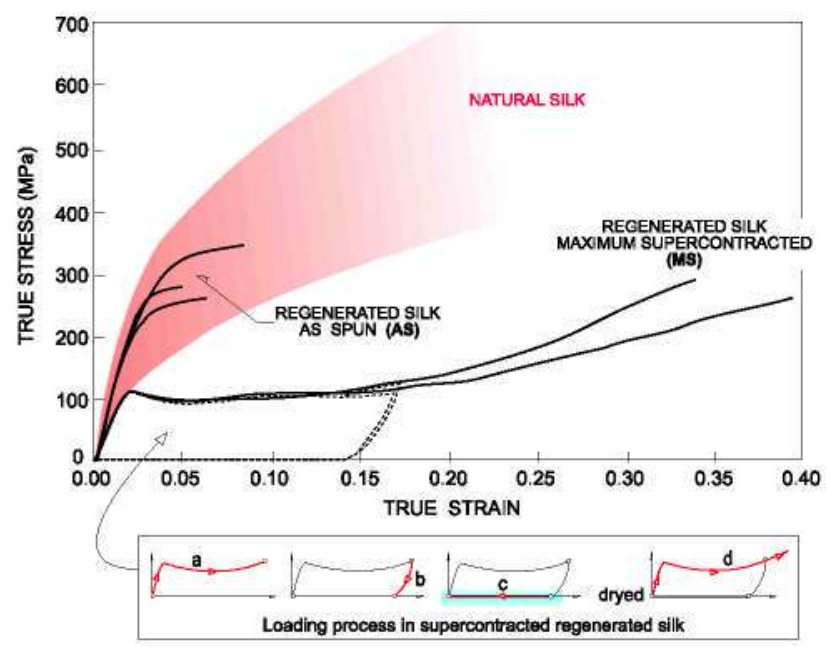

Fig. 10 - Stress-strain curves of immersion post spinning drawing (IPSD) fibers under the as-spun condition (AS) and after maximum supercontraction (MS). The range of curves from natural Bombyx mori silk fibers is shown to allow comparison. The dotted lines correspond to a recovery test on the previously supercontracted fibers.

Amino acid analysis showed that regenerated and native spider silks were similar in composition. On the contrary, the mechanical properties of regenerated silk filaments were very poor in comparison with natural silk fibers, although the extensibility - around $30 \%$ - was similar to native spider silk. The authors conjecture was that the relatively poor mechanical properties of the regenerated silk was due to its formation by a self-assembling process of molecular chain aggregation, instead of the complex liquid crystal spinning of native silk. Thus, the unique mechanical behavior of spider silk is controlled not only by silk protein gene, but as much by the processes in which these are assembled into the silk fibers.

Processing of regenerated silkworm silk can also shed some light on this subject. Regenerated silk fibroin solutions from Bombyx mori were spun using an extrusion unit, a coagulation bath, a take-up device, and an immersion post spinning drawing (IPSD), as described elsewhere (Plaza et al., 2008, 2009).

Fig. 10 shows the stress-strain curves of regenerated silkworm fibers spun according the IPSD procedure. The range of stress-strain curves of natural silkworm silk fibers (Pérez-Rigueiro et al., 1998) is also indicated. The elastic modulus of the as-spun fibers lies within the range observed in natural fibers. Although the tensile strength and the strain at breaking improve significantly compared with previous values of fibers subjected to post spinning drawing in air, they still lie below those of the natural material. Consequently, the work of fracture does not reach the outstanding values of natural silkworm silk.

In addition, Fig. 10 shows the tensile properties of IPSD fibers subjected to supercontraction. The existence of a true maximum supercontacted state in IPSD fibers is shown by the recovery of the fibers, also illustrated in Fig. 10. The concurrence among the original maximum supercontracted curves prior to and after the first stretching step, indicates that the IPSD fibers can recover from irreversible deformations and that there is a ground state that can be reached independently 
of the previous loading history of the fiber. Therefore, the effect of water on the unrestrained IPSD fibers can be labeled properly as supercontraction (Elices et al., 2004).

These results show the ability of IPSD fibers to recover from irreversible deformation and that their tensile behavior can be tailored repeatedly; features solely exhibited until now by natural spider silk. As a result, it would make it possible to control their molecular alignment by methods similar to those applied to spider silk (Guinea et al., 2005b), and lead to different mechanical behavior in a repeatable and accurate procedure. This finding demonstrates that processing plays a role at least comparable to that of the amino acid sequence in the final properties of the material.

\subsection{Processing of genetically engineered analogues of spider silk}

As already mentioned, biotechnological production of spider silk is not only a promising altemative to mass production, but also one that enables evaluation of the role of composition by spinning dopes of silk-like proteins based on sequences of dragline silk proteins.

Several studies have investigated spinning of artificial dopes from engineered spider silk proteins (Lazaris et al., 2002; Hardy et al., 2008; Rammensee et al., 2008). Although very few silk genes have been completely cloned (Xia et al., 2004; Ayoub et al., 2007), due to their large size (Xu and Lewis, 1990), the existence of a small number of simple motifs of sequence extensively repeated (Gatesy et al., 2001) has led to the synthesis of artificial analogs that are believed to capture the essential features of the natural proteins, though so far no process has resulted in silk fibers that perfectly mimic the mechanical properties of natural silks.

A review of the mechanical performance of silk fibers spun from artificial dopes can be viewed in the quoted references. Our aim here is to highlight the role of supercontraction on the tensile behavior of these artificial silks. To this end, some recent results by the authors are briefly summarized.

Two polypeptides have been used that include the repetitive motifs of sequence of the major ampullate silk proteins of the spider $N$. clavipes (Gatesy et al., 2001). The polypeptides were labeled as $\mathrm{rcSp} 1$ and $\mathrm{rcSp} 2$, referring to the spidroin on which are inspired (Xu and Lewis, 1990). The production of the recombinant proteins, preparation of the spin dopes and spinning of the fibers are described in detail elsewhere (Karatzas et al., 2005).

Fig. 11 shows the mechanical properties of recombinant silk fibers of the two compositions as spun. The tensile strength is still lower than the tensile strength of natural spider silk fibers, while the elongation at break lies in the range of those fibers (Garrido et al., 2002a,b). From these results it is apparent that the differences in composition do not exert a significant influence (unless with the used spinning procedure) on the mechanical properties of as-spun recombinant fibers tested in air.

The extent of supercontraction in recombinant fibers has been analyzed by measuring the reduction in length of unrestrained fibers in two steps: measurement of the reduction in length after immersion in water and measurement of the reduction in length following drying. These recombinant fibers display all the effects customarily associated with supercontraction and the existence of a

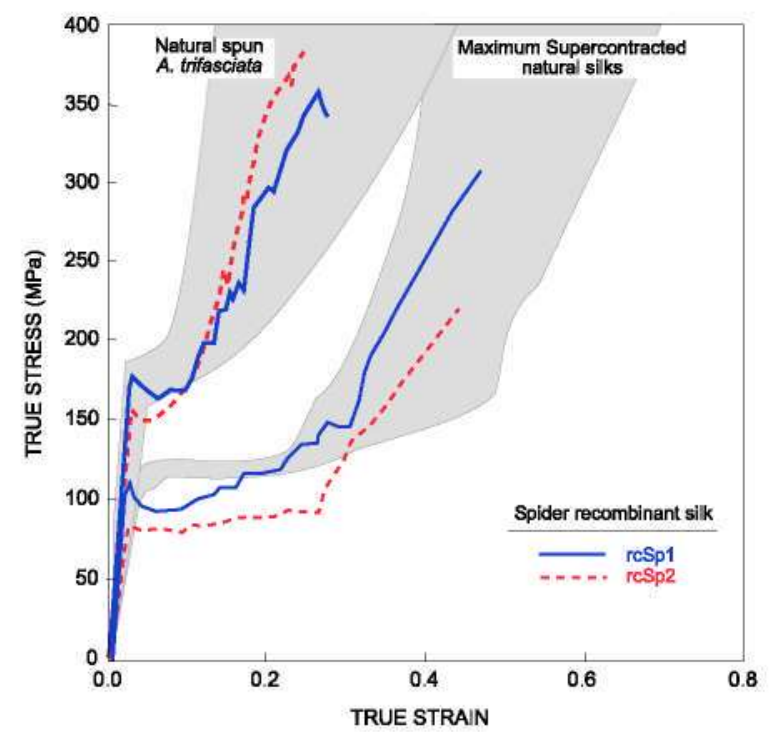

Fig. 11 - Stress-strain curves of recombinant silk fibers: in the as-spun condition and after maximum supercontraction. In both examples the initial range of values of natural spider silks is also shown.

ground state - recovery from irreversible stretching in air, the possibility of modifying the properties predictably and reproducibly by stretching them, and their elastomeric behavior in water -, the values of supercontraction are relatively modest, varying from $16 \%-24 \%$, and far from the values of natural spider silk fibers (Elices et al., 2009).

Fig. 11 shows the tensile properties of maximum supercontracted (MS) fibers tested in air. As observed with the fibers in the as-spun condition, MS fibers show comparable tensile properties, essentially independent of their composition. Compared with natural spider silk, recombinant MS fibers present a lower tensile strength (210-280 MPa), and lower elongation at breaking $(0.4-0.5)$. Furthermore, the yield point at the end of the elastic region is slightly lower in recombinant fibers.

These findings suggest that it is possible to produce high performance fibers from recombinant proteins with a conventional wet spinning process. That exact composition of the dope is not essential for the tensile behavior of the fibers, although recombinant fibers show tensile strengths significantly below those of natural MA silk and, more important for the purpose of this paper, that recombinant fibers exhibit a ground state to which the fiber can revert independently of its previous loading history. The assessment of the existence of supercontraction in bio-inspired fibers has significant implications for design (as fibers can be custom tailored) and for practical use (due to desirable properties, such as recovery and adaptability, conferred to the fiber).

REFERENCES

Ayoub, N.A., Garb, J.E., Tinghitella, R.M., Collin, M.A., Hayashi, C.Y., 2007. Blueprint for a high-performance biomaterial: full-length spider dragline silk genes. PLoS ONE 6, e514.

Bell, F.I., McEwen, I.J., Viney, C., 2002. Fibre science. Supercontraction stress in wet spider dragline. Nature 416, 37. 
Denny, M.W., 1976. The physical properties of spider's silk and their role in the design of orb-webs. J. Exp. Biol. 65, 483-506.

Elices, M., Pérez-Rigueiro, J., Plaza, G., Gninea, G.V., 2004. Recovery in spider silk fibers. J. Appl. Polym. Sci. 92, 3537-3541.

Elices, M., Pérez-Rigueiro, J., Plaza, G., Gninea, G.V., 2005. Finding inspiration in A. trifasciata spider silk fibers. JOM 2, 60-66.

Elices, M., Plaza, G.R., Arnedo, M.A., Pérez-Rigueiro, J., Torres, F.G. Gninea, G.V., 2009. The mechanical behavionr of silk dnring the evolntion of orb-web spinning spiders. Biomactomolecules 10 , 1904-1910.

Ganido, M.A., Elices, M., Viney, C., Pérez-Rigueiro, J., 2002a. Active control of spider silk strength: comparison of drag line spun on vertical and horizontal surfaces. Polymer 43, 1537-1540.

Ganido, M.A., Elices, M., Viney, C., Pérez-Rigueiro, J., 2002b. The variability and interdependence of spider drag line tensile properties. Polymer 43, 4495-4502.

Gatesy, J., Hayashi, C., Motrink, D., Woods, J., Lewis, R., 2001. Extreme diversity, conservation and convergence of spider silk fibroin sequences. Science 291, 2603-2605.

Gosline, J.M., Denny, M.W., Demont, M.E., 1984. Spider silk as rubber. Natnre 309, 551-552.

Gosline, J.M., Guerette, P.A., Ortilepp, C.S., Savage, K.N., 1999. The mechanical design of spider silks: from fibroin sequence to mechanical function. J. Exp. Biol. 202, 3295-3303.

Gosline, J.M., Pollak, C.C., Guerette, P.A., Cheng, A., Demont, M.E., Denny, M.W., 1994. Elastomeric network models for the frame and viscid silks from the orb web of the spider Araneus-Diadematus. Silk. Polymers 544, 328-341.

Gninea, G.V., Elices, M., Pérez-Rigueiro, J., Plaza, G., 2003. Selftightening of spider silk fibers induced by moistnre. Polymer 44, 5785-5788.

Guinea, G.V., Elices, M., Real, J., Gutiérrez, S., Pérez-Rigueiro, J., 2005a. Reproducibility of the tensile properties of spider (A. trifasciata) silk obtained by forced silking. J. Exp. Zool. 303A, $37-44$.

Guinea, G.V., Elices, M., Pérez-Rigueiro, J., Plaza, G.R., 2005b. Stretching of supercontracted fbers: a link between spinning and the variability of spider silk. J. Exp. Biol. 208, 25-30.

Hardy, J.G., Romer, L.M., Scheibel, T.R., 2008. Polymeric materials based on silk proteins. Polymer 49, 4309-4327.

Hayashi, C.Y., Shipley, N.H., Lewis, R.V., 1999. Hypothesis that correlate the sequences, structure, and mechanical properties of spider silk proteins. Int. J. Biol. Macromol. 24, 271-275.

Heim, M., Keerl, D., Scheibel, T., 2009. Spider silk: from soluble protein to extraordinary fiber. Angew. Chem. Int. Ed. 48, 2-15.

Jelinski, L.W., Blye, A., Liivak, O., Michal, C., LaVerde, G., Seidel, A., Shah, N., Yang, Z., 1999. Orientation, structure, wet-spinning, and molecular basis for supercontraction of spider silk. [nt. ). Biol. Macromol. 24, 197-201.

Kaplan, D., Adams, W., Farmer, B., Viney, C. (Eds.), 1994. Silk Polymers. In: Am. Chem. Society Symp. Series, vol. 544.

Kaplan, D.L., Lombardi, S., Muller, W.S., Fossey, S.A., 1991. Biomaterials. Novel Materials from Biological Sources. Stockon Press, New York, pp. 53

Karatzas, C.N., Chrétien, N., Dngnay, F., Bellemare, A., Zhou, J.F., Rodenhiser, A., Islam, S.A., Turcotte, C., Hnang, Y., Lazaris, A, 2005. High-toughness spider silk fibers spun from soluble recombinant silk produced in mammalian cells. In: Steinbüchel, Doi (Eds.), Biotechnology of Biopolymers, vol. 2. Wiley-VCH, Weinheim, Pp. 945-967 (Chapter 33).

Kluge, J.A., Rabotyagora, O., Leisk, G., Kaplan, D.L., 2008. Spider silks and their applications. Trends Biotechnol. 26, 244-251

Lazaris, A., Arcidiacono, S., Hnang, Y., Zhou, J.F., Duguay, F., Chretien, N., Welsh, E.A., Soares, J.W., Karatzas, C.N., 2002. Spider silk fibers spun from soluble recombinant silk produced in mammalian cells. Science $295,472-476$.

Lewis, R.V., 1992. Spider silk: the nnraveling of a mystery. Acc. Chem. Res. 25, 392-398.
Lin, Y., Shao, Z., Vollrath, F., 2005. Relationships between supercontraction and mechanical properties of spider silk. Nat. Mater. 4, $901-905$

Madsen, B., Shao, Z.Z., Vollrath, F., 1999. Variability in the mechanical properties of spider silks on three levels: interspecific, intraspecific and intraindividual. Int. J. Biol. Macromol. 24, 301-306.

O'Brien, J.P., Fahnestock, S.R., Termonia, Y., Gardner, K.H., 1998 Nylons from nature. Synthetic analogs to spider silk. Adv Mater. 10, 1185-1195.

Pérez-Rigueiro, J., Elices, M., Guinea, G.V., 2003. Controlled snpercontraction tailors the tensile behaviour of spider silk Polymer 44, 3733-3736.

Pérez-Rigueiro, J., Elices, M., Llorca, J., Viney, C., 2001. Tensile properties of A. trifasciata drag line silk obtained from the spider's web. J. Appl. Polym. Sci. 82, 2245-2251.

Pérez-Rigueiro, J., Elices, M., Plaza, G., Real, J.I., Gninea, G.V., 2005 The effect of spinning forces on spider silk proteins. J. Exp. Biol. $28,2633-2639$.

Pérez-Rigueiro, J., Viney, C., Llorca, J., Elices, M., 1998. Silkworm silk as an engineering material. J. Appl. Polym. Sci. 70, 2439-2447.

Pérez-Rigueiro, J., Viney, C., LJorca, J., Elices, M., 2000. Mechanical properties of single-brin silkworm silk. J. Appl. Polym. Sci. 75, $1270-1277$

Planas, J., Guinea, G.V., Elices, M., 2007. Constitutive model for fiber-reinforced materials with deformable matrices. Phys. Rev. E 76, 041903

Plaza, G.R., Corsini, P., Marsano, E., Pérez-Rigueiro, J., Biancotto, L., Elices, M., Riekel, C., Agulló-Rueda, F., Gallardo, E., Calleja, I.M., Guinea, G.V., 2009. Old silks endowed with new properties. Macromolecules 42, 8977-8982.

Plaza, G.R., Corsini, P., Pérez-Rigneiro, J., Marsano, E., Gninea, G.V., Elices, M., 2008. Effect of water on Bombyx mori regenerated silk fibers and its application in modifying their mechanical properties. J. Appl. Polym. Sci. 109, 1793-1801.

Plaza, G.R., Guinea, G.V., Pérez-Rigueiro, I., Elices, M., 2006 Thermo-hygro-mechanical behavior of spider dragline silk glassy and rubbery states. J. Polym. Sci. B 44, $994-999$.

Rammensee, S., Slotta, U., Scheibel, T., Bausch, A.R., 2008. Assembly mechanism of recombinant spider silk proteins Proc. Natl. Acad. Sci. USA 105, 6590-6595

Savage, K.N., Guerette, P.A., Gosline, J.M., 2004. Supercontraction stress in spider webs. Biomacromolecules 5, 675-679.

Seidel, A., Lijvak, O., Calve, S., Adeska, J, Ji, G., Yank, Z., Grubb, D., Zax, D., Jelinski, L.W., 2000. Regenerated spider silk: processing, properties and structnre. Macromolecules 33, 775-780.

Shao, Z., Vollrath, F., Yang, Y., Thøgersen, H.C., 2003. Structure and behavior of regenerated spider silk. Macromolecnles 36, 1157-1161.

Swanson, B.O., Blackledge, T.A., Summers, A.P., Hayashi, C.Y., 2006. Spider dragline silk: correlated and mosaic evolution in high-performance biological materials. Evolntion 60 (12), 2539-2551.

Termonia, Y., 1994. Molecular modeling of spider silk elasticity. Macromolecules 27, 7378-7381.

Termonia, Y., 2000. Molecnlar modeling of the stress/strain behavionr of spider dragline. In: Elices, M. (Ed.), Structnral Biological Materials. Pergamon Press, Amsterdam, pp. 335-349.

Viney, $C ., 2000$. Silk fibres: origins, nature and consequences of structre. In: Elices, M. (Ed.), Structnral Biological Materials. Pergamon Press, Amsterdam, pp. 293-333.

Vollrath, F., 1992. Spider webs and silks. Sci. Am. 266, 70-76.

Vollrath, F, Knight, D.P., 2001. Liquid crystalline spinning of spider silk. Nature 410, 541-548.

Weidmann, G., Lewis, P., Reid, N., 1990. Fibres and fibre assemblies. In: Structural Materials. Butterworths The Open University, (Chapter 6) 
Work, R.W., 1976. The force elongation behaviour of web fibers and silks forcibly obtained from orb-web-spinning spiders. Text. Res. J. 46, 485-492.

Work, R.W., 1977. Dimensions, birefringences, and forceelongation behavior of major and minor ampullate silk fibers from orb-web-spinning spiders-effects of wetting on these properties. Text. Res. J. 47, 650-662.

Work, R.W., 1981. A comparative study of the supercontraction of major ampullate silk tibers of orb-web-building spiders. J. Arachnol. 9, 299-308.
Work, R.W., 1985. Viscoelastic behavior and wet snpercontraction of major ampullate silk fibres of certain orb-web-bnilding spiders (Aranae). J. Exp. Biol. 118, 379-404.

Xia, Q.Y, Zhou, Z.Y, Lu, C., Cheng, D.], Dai, F.Y., Li, B., Zhao, P., Zha, X.F., Cheng, T.C., Chai, C.L., et al., 2004. A draft sequence for the genome of the domesticated silkworm (Bombyx mori). Science 306, 1937-1940.

Xu, M., Lewis, R.V., 1990. Structure of a protein superfiber-spider dragline silk. Proc. Natl. Acad. Sci. USA 87, 7120-7124. 